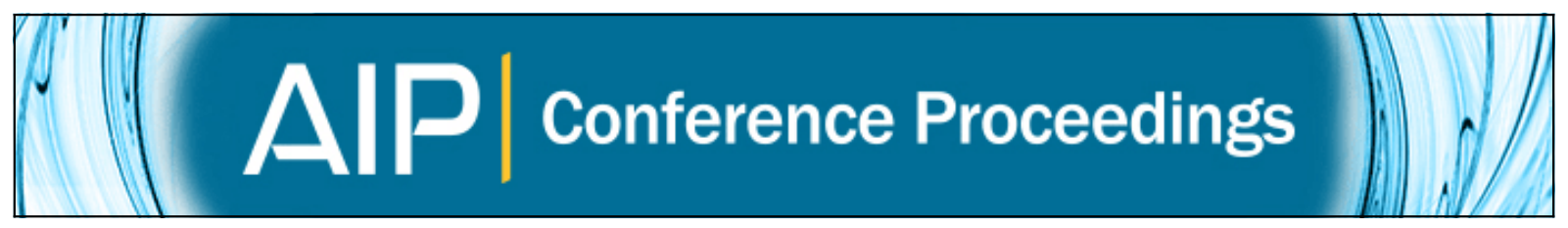

\title{
Discrete hypercomplex function theory and its applications
}

Uwe Kähler

Citation: AIP Conference Proceedings 1648, 020012 (2015); doi: 10.1063/1.4912316

View online: http://dx.doi.org/10.1063/1.4912316

View Table of Contents: http://scitation.aip.org/content/aip/proceeding/aipcp/1648?ver=pdfcov

Published by the AIP Publishing

Articles you may be interested in

Complex and Hypercomplex Methods in Applications

AIP Conf. Proc. 1048, 627 (2008); 10.1063/1.2991004

Applications of Hyper-Complex Analysis in Yang-Mills Gauge Theories

AIP Conf. Proc. 936, 754 (2007); 10.1063/1.2790262

Dynamical density functional theory and its application to spinodal decomposition

J. Chem. Phys. 121, 4246 (2004); 10.1063/1.1778374

Response function theory and its application to polymer dynamics

J. Chem. Phys. 79, 1013 (1983); 10.1063/1.445842

A Property of Bessel Functions and Its Application to the Theory of Two Rheometers

J. Appl. Phys. 23, 1070 (1952); 10.1063/1.1701988 


\title{
Discrete Hypercomplex Function Theory and its Applications
}

\author{
Uwe Kähler \\ Departamento de Matemática, Universidade de Aveiro, P-3810-193 Aveiro, Portugal
}

\begin{abstract}
Recently, one can observe an increased interest in discrete function theories and their applications. Although we will give a broader overview in our talk we would like to give a closer idea on the topic and its applications. To this end we present the question of boundary values of discrete monogenic functions in this short text. We also show their applicability in the theory of discrete Riemann boundary value problems (Riemann BVP's). The grid itself was chosen in view of applications to image processing, such as discrete monogenic functions.
\end{abstract}

Keywords: Discrete Dirac operator, Discrete Hardy spaces, Discrete Riemann boundary value problems PACS: $02.30 . \mathrm{Em}, 02.30 . \mathrm{Fn}, 02.30 . \mathrm{Nw}, 02.70 . \mathrm{Bf}$

\section{PRELIMINARIES}

Let us begin with some basic facts of discrete function theory. Without loss of generality we restrict ourselves to the (for practical applications) most important case $d=3$.

Consider the grid $h \mathbb{Z}^{3}$, with orthonormal basis $e_{k}, k=1,2,3$, with $h>0$ being the lattice constant (mesh size) and the standard forward and backward differences $\partial_{h}^{ \pm j}$ given by

$$
\partial_{h}^{+j} f(m h)=h^{-1}\left(f\left(m h+e_{j} h\right)-f(m h)\right), \quad \partial_{h}^{-j} f(m h)=h^{-1}\left(f(m h)-f\left(m h-e_{j} h\right)\right),
$$

for $h m=h\left(m_{1} e_{1}+m_{2} e_{2}+m_{3} e_{3}\right) \in h \mathbb{Z}^{3}$. For a discrete function theory we introduce a discrete Dirac operator which factorizes the star-Laplacian $\Delta_{h}$ by splitting each basis element $e_{k}, k=1,2,3$, into two basis elements $e_{k}^{+}$and $e_{k}^{-}, k=1,2,3$, i.e., $e_{k}=e_{k}^{+}+e_{k}^{-}, k=1,2,3$, corresponding to the forward and backward directions. The new basis elements should satisfying the following multiplication relations:

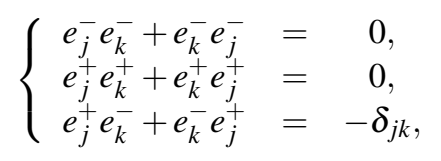

where $\delta_{j k}$ is the Kronecker delta. These basis elements $\left\{e_{1}^{ \pm}, e_{2}^{ \pm}, e_{3}^{ \pm}\right\}$generate the complexified Clifford algebra $\mathbb{C}_{3}=\mathbb{C} \otimes_{\mathbb{R}} \mathbb{R}_{0,3}$. Keep in mind that the norm is no longer preserved under multiplication. In fact, we have only the estimate (see [3])

$$
|a b| \leq 2^{3}|a||b|, \quad a, b \in \mathbb{C}_{3} .
$$

Furthermore, we consider functions defined on $(\emptyset \neq) G \subset h \mathbb{Z}^{3}$ and taking values in $\mathbb{C}_{3}$. Properties like $l_{p^{-}}$ summability $(1 \leq p<\infty)$ and so on, are defined for a $\mathbb{C}_{3}$-valued function by being ascribed to each component. The corresponding spaces are denoted, respectively, by $l_{p}\left(G, \mathbb{C}_{3}\right),(1 \leq p<+\infty)$ and so on.

The discrete Dirac operator $D^{+-}$and its adjoint operator $D^{-+}$are defined by

$$
D_{h}^{+-}=\sum_{j=1}^{3} e_{j}^{+} \partial_{h}^{+j}+e_{j}^{-} \partial_{h}^{-j}, \quad D_{h}^{-+}=\sum_{j=1}^{3} e_{j}^{+} \partial_{h}^{-j}+e_{j}^{-} \partial_{h}^{+j} .
$$

Each operator factorizes the star-Laplacian (a self-adjoint operator)

$$
\Delta_{h}=\sum_{j=1}^{3} \partial_{h}^{+j} \partial_{\mathbf{h}}^{-\mathbf{j}}
$$

i.e.,

$$
\left(D_{h}^{+-}\right)^{2}=\left(D_{h}^{-+}\right)^{2}=-\Delta_{h} .
$$


This idea is different from the case of discrete complex analysis [6]. For more details we refer the reader to the literature, e.g. [1],[5],[3],[4].

\section{DISCRETE BOUNDARY VALUES}

The principal difference between discrete and continuous boundary values is that in the first ones are defined on two boundary layers while only one layer is enough in the second case. This is a direct consequence of having two difference operators (forward and backward differences) instead of a single derivative.

Here we present the following two theorems 1 and 3, where the boundary value of discrete monogenic functions in the upper, resp. lower, lattice is characterized in terms of their behaviour in the Fourier domain, as well as their corollaries, which provided us with closed formulae for the symbols of the discrete Hilbert transforms in the upper, resp. the lower, lattice. The proofs can be found in [1].

Theorem 1 Let $f \in l_{p}\left(h \mathbb{Z}^{2}, \mathbb{C}_{3}\right)$ given by $f=f_{1}+e_{3}^{+} f_{2}+e_{3}^{-} f_{3}+e_{3}^{+} e_{3}^{-} f_{4}$, with $f_{i}: h \mathbb{Z}^{2} \rightarrow \mathbb{C}_{2}, i=1,2,3,4$. Then $f$ is the boundary value of a discrete monogenic function in the discrete upper half plane if and only if its discrete $2 D$-Fourier transform $F=\mathscr{F}_{h} f$, with

$$
F(\underline{\xi})=F_{1}(\underline{\xi})+e_{3}^{+} F_{2}(\underline{\xi})+e_{3}^{-} F_{3}(\underline{\xi})+e_{3}^{+} e_{3}^{-} F_{4}(\underline{\xi}), \quad \underline{\xi} \in\left[-\frac{\pi}{h}, \frac{\pi}{h}\right]^{2},
$$

satisfies the system

$$
\left\{\begin{array}{c}
\frac{h \underline{d}-\sqrt{4+h^{2} \underline{d}^{2}}}{2} F_{1}+\frac{\widetilde{\xi}_{-}}{\underline{d}} F_{2}=0, \\
\frac{h \underline{d}-\sqrt{4+h^{2} \underline{d}^{2}}}{2} F_{3}+\frac{\widetilde{\xi}}{\underline{d}}-\left(F_{1}-F_{4}\right)=0
\end{array}\right.
$$

Corollary 2 Let $f \in l_{p}\left(h \mathbb{Z}^{2}, \mathbb{C}_{3}\right)$ be a boundary value of a discrete monogenic function in the upper half space. Then its $2 D$-Fourier transform $F=\mathscr{F} h$, satisfies the equation

$$
\frac{\underline{\tilde{\xi}}}{\underline{d}}\left(e_{3}^{+} \frac{h \underline{d}-\sqrt{4+h^{2} \underline{d}^{2}}}{2}+e_{3}^{-} \frac{2}{h \underline{d}-\sqrt{4+h^{2} \underline{d}^{2}}}\right) F=F .
$$

Theorem 3 Let $f \in l_{p}\left(h \mathbb{Z}^{2}, \mathbb{C}_{3}\right)$ given by $f=f_{1}+e_{3}^{+} f_{2}+e_{3}^{-} f_{3}+e_{3}^{+} e_{3}^{-} f_{4}$, with $f_{i}: h \mathbb{Z}^{2} \rightarrow \mathbb{C}_{2}, i=1,2,3,4$. Then $f$ is the boundary value of a discrete monogenic function in the discrete lower half plane if and only if its $2 D$-Fourier transform $F=\mathscr{F}_{h} f$, with

$$
F(\underline{\xi})=F_{1}(\underline{\xi})+e_{3}^{+} F_{2}(\underline{\xi})+e_{3}^{-} F_{3}(\underline{\xi})+e_{3}^{+} e_{3}^{-} F_{4}(\underline{\xi}), \quad \underline{\xi} \in\left[-\frac{\pi}{h}, \frac{\pi}{h}\right]^{2},
$$

satisfies the system

$$
\left\{\begin{array}{c}
\frac{h \underline{d}-\sqrt{4+h^{2} \underline{d}^{2}}}{2} F_{2}-\frac{\tilde{\xi}_{-}}{\underline{d}} F_{1}=0, \\
\frac{h \underline{d}-\sqrt{4+h^{2} \underline{d}^{2}}}{2}\left(F_{1}-F_{4}\right)-\frac{\underline{\tilde{\xi}}_{-}}{\underline{d}} F_{3}=0 .
\end{array}\right.
$$

Corollary 4 Let $f \in l_{p}\left(h \mathbb{Z}^{2}, \mathbb{C}_{3}\right)$ be a boundary value of a discrete monogenic function in the lower half space. Then its $2 D$-Fourier transform $F=\mathscr{F}_{h} f$, satisfies the equation

$$
-\frac{\underline{\xi}}{\underline{d}}\left(e_{3}^{+} \frac{2}{h \underline{d}-\sqrt{4+h^{2} \underline{d}^{2}}}+e_{3}^{-} \frac{h \underline{d}-\sqrt{4+h^{2} \underline{d}^{2}}}{2}\right) F=F .
$$

Both theorems and their corollaries allow us to introduce upper and lower discrete Hardy spaces as the space of functions which fulfill (2) and (3), respectively.

Since we have two boundary layers one could think that the discrete boundary values are independent on both layers. This is not the case as can be seen in the next theorem where we introduce the operators $A^{+}, A^{-}$, which reconstruct the 
boundary data in the 0 -layer from the knowledge of boundary data in the 1-layer, resp. -1 -layer, thus giving the complete boundary data of a function in the upper, resp. lower, discrete Hardy space.

Theorem 5 An arbitrary function $f \in l_{p}\left(\mathbb{Z}^{2}, \mathbb{C}_{3}\right)$ can be decomposed into a pair of functions $P_{+} f$ and $Q_{+} f$ where $P_{+} f \in h_{p}^{+}$, i.e. it can be extended to the zero layer via its action in the Fourier domain by

$$
e_{3}^{-} F^{+, 0}=\frac{\widetilde{\xi}_{-}}{\underline{d}} \frac{h \underline{d}+\sqrt{4+h^{2} \underline{d}^{2}}}{2}\left(-e_{3}^{-} F_{1}^{+, 1}+e_{3}^{-} e_{3}^{+} F_{3}^{+, 1}\right):=A^{+} F^{+, 1},
$$

with $F^{+, 1}=\mathscr{F}_{h} f$ and $F^{+, 1}$ fulfills (2). In the same way an arbitrary function $f \in l_{p}\left(\mathbb{Z}^{2}, \mathbb{C}_{3}\right)$ can be decomposed into a pair of functions $P_{-} f$ and $Q_{-} f$ where $P_{-} f \in h_{p}^{-}$, i.e. it can be extended to the zero layer via its action in the Fourier domain by

$$
\begin{aligned}
e_{3}^{+} F^{-, 0}= & \frac{2 \sqrt{4+h^{2} \underline{d}^{2}}}{h \underline{d}+\sqrt{4+h^{2} \underline{d}^{2}}}\left(-e_{3}^{+} F_{1}^{-,-1}-e_{3}^{+} e_{3}^{-} F_{3}^{-,-1}\right) \\
& +\frac{\underline{\underline{\xi}}}{\underline{d}}\left(\frac{h^{2} \underline{d}^{2}-1-h \underline{d} \sqrt{4+h^{2} \underline{d}^{2}}}{h \underline{d}-\sqrt{4+h^{2} \underline{d}^{2}}}\right)\left(e_{3}^{+} F_{2}^{-,-1}-e_{3}^{+} e_{3}^{-}\left(F_{1}^{-,-1}-F_{4}^{-,-1}\right)\right):=A^{-} F^{-,-1}
\end{aligned}
$$

with $F^{-,-1}=\mathscr{F}_{h} f$ and $F^{-,-1}$ fulfills (3).

\section{DISCRETE RIEMANN BVP}

The above considerations allow us to study discrete Riemann boundary value problems. Two examples are given below. The proofs can be found in [2] although they are essentially an adaptation of the classic proofs using the results from the previous section.

Theorem 6 The problem

$$
\begin{array}{ccc}
D_{h}^{-+} f(n) & = & 0, n \in \mathbb{Z}^{3} \backslash\left\{n_{3}=0\right\}, \\
f(\underline{n}, 1) & = & g(\underline{n}), \underline{n} \in \mathbb{Z}^{2} .
\end{array}
$$

with $g \in h_{p}^{+}\left(\mathbb{Z}^{2}\right)$ has a unique solution given by the respective Hardy/Plemelj-projections.

Theorem 7 The problem

$$
\begin{array}{ccc}
D_{h}^{-+} f(n) & = & 0, n \in \mathbb{Z}^{3} \backslash\left\{n_{3}=0\right\}, \\
A^{+}(f(\underline{n}, 1)) & = & A^{-}(f(\underline{n},-1))+g(\underline{n}), \underline{n} \in \mathbb{Z}^{2} .
\end{array}
$$

with $g \in l_{p}\left(\mathbb{Z}^{2}\right)$ has a unique solution given by the respective Hardy/Plemelj-projections.

\section{ACKNOWLEDGMENTS}

This work was supported by Portuguese funds through the CIDMA - Center for Research and Development in Mathematics and Applications, and the Portuguese Foundation for Science and Technology ("FCT-Fundação para a Ciência e a Tecnologia"), within project PEst-OE/MAT/UI4106/2014.

\section{REFERENCES}

1. P. Cerejeiras, U. Kähler, M. Ku, F. Sommen, Discrete Hardy Spaces, Journal of Fourier Analysis and Applications, 20, 2014, 715-750.

2. P. Cerejeiras, U. Kähler, M. Ku, Discrete Riemann-Hilbert boundary value problems in higher dimensions, in preparation.

3. R. Delanghe, F. Sommen, V. Soucek, Clifford Algebra and Spinor-Valued Functions, Kluwer Academic, Dordrecht, 1992.

4. H. De Ridder, H. De Schepper, U. Kähler, F. Sommen, Discrete function theory based on skew Weyl relations, Proceeding of the American Mathematical Society, 138(9), 2010, 3241-3256.

5. K. Gürlebeck, W. Sprößig, Quaternionic and Clifford calculus for physicists and engineers, Wiley, 1997.

6. L. Lovasz, Discrete Analytic Functions: An Exposition, Surveys in differential geometry, Vol. IX (2004), 1-44. 